\title{
Spatial distribution of accreting isolated neutron stars in the Galaxy
}

\author{
S.B.Popov and M.E.Prokhorov \\ Sternberg Astronomical Institute, Moscow State University
}

\begin{abstract}
We present here the computer model of the distribution of the luminosity, produced by old isolated neutron stars (OINSs) accreting from the interstellar medium (ISM). We show, that for different mean velocities of OINSs the distribution of the luminosity has a torus-like structure, with the maximum at $\approx 5 k p c$.
\end{abstract}

\section{Introduction}

In the last several years, the spatial distribution of old isolated neutron stars (OINSs) became of great interest (see, for example, Treves and Colpi (1991)). Several sources of this size were observed by ROSAT. Different regimes of interaction of the interstellar medium (ISM) and OINSs can appear: Ejector, Propeller (with possible transient source), Accretor, Georotator and supercritical regimes (see, Popov (1994) and Lipunov and Popov (1995)). Here we are interested only in accreting OINSs.

We use direct calculations of trajectories in the Galaxy potential, taken in the form (Paczynski (1990)):

$$
\Phi_{i}(R, Z)=G M_{i} /\left(R^{2}+\left[a_{i}+\left(Z^{2}+b_{i}^{2}\right)^{1 / 2}\right]^{2}\right)^{1 / 2}
$$

In the articles of Postnov and Prokhorov $(1993,1994)$ it was shown, that OINSs in the Galaxy form a torus-like structure. If one looks at their distribution and at the distribution of the ISM (see, for example, Bochkarev (1993) ), it is clearly seen, that the maximums of two distributions roughly coincides. It means, that most part of OINSs is situated in dence regions of ISM. So, the luminosity there must be higher. Here we represent computer simulations of this situation.

\section{Model}

We made calculations on the grid with the cell size $100 \mathrm{pc}$ in R-direction and 10 $\mathrm{pc}$ in $\mathrm{Z}$-direction (centered at $\mathrm{R}=50 \mathrm{pc}, \mathrm{Z}=5 \mathrm{pc}$ and so on). Stars were born in the Galactic plane. The system of differential equations was solved numerically.

In our model we assumed, that the birthrate of NSs is proportional to the square of local density. Local density was calculated using data and formulaes from Bochkarev (1993) and Zane et al. (1995). 


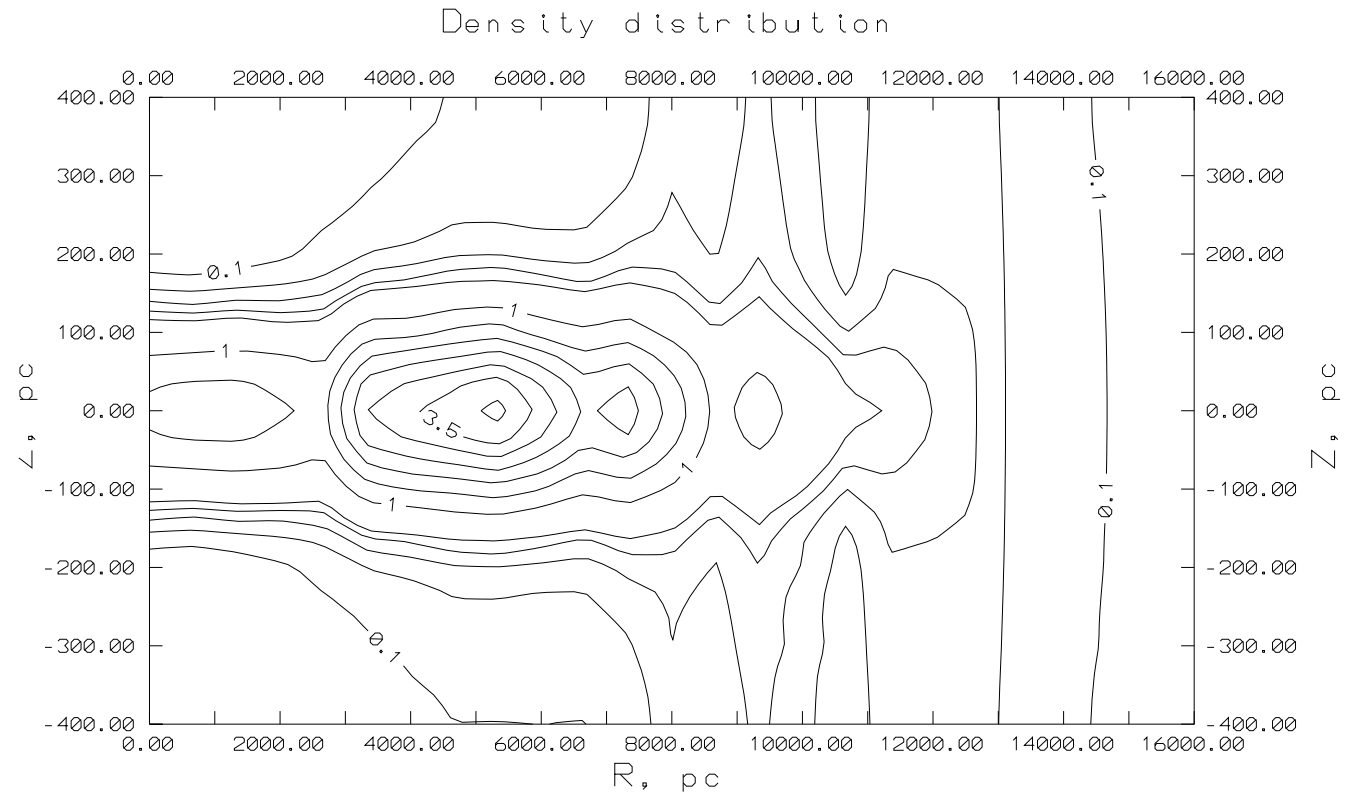

Figure 1: The density distribution in R-Z plane

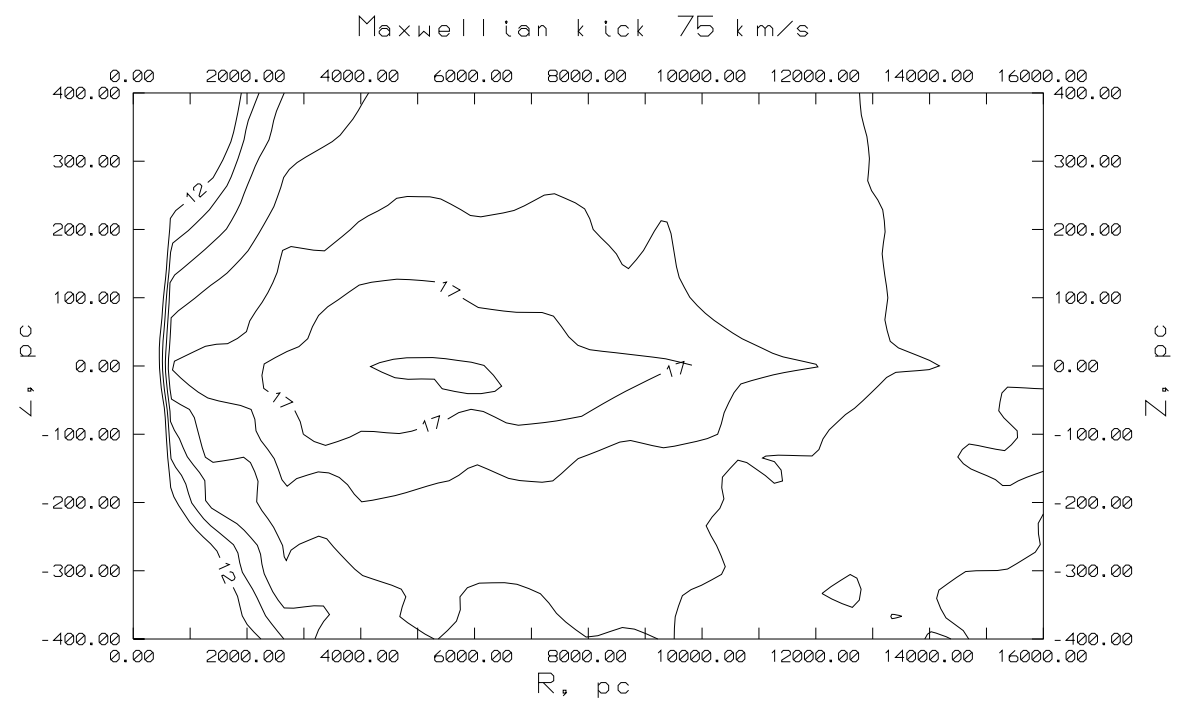

Figure 2: The luminosity distribution in R-Z plane for Maxwellian kick velocity $(75 \mathrm{~km} / \mathrm{s})$ 


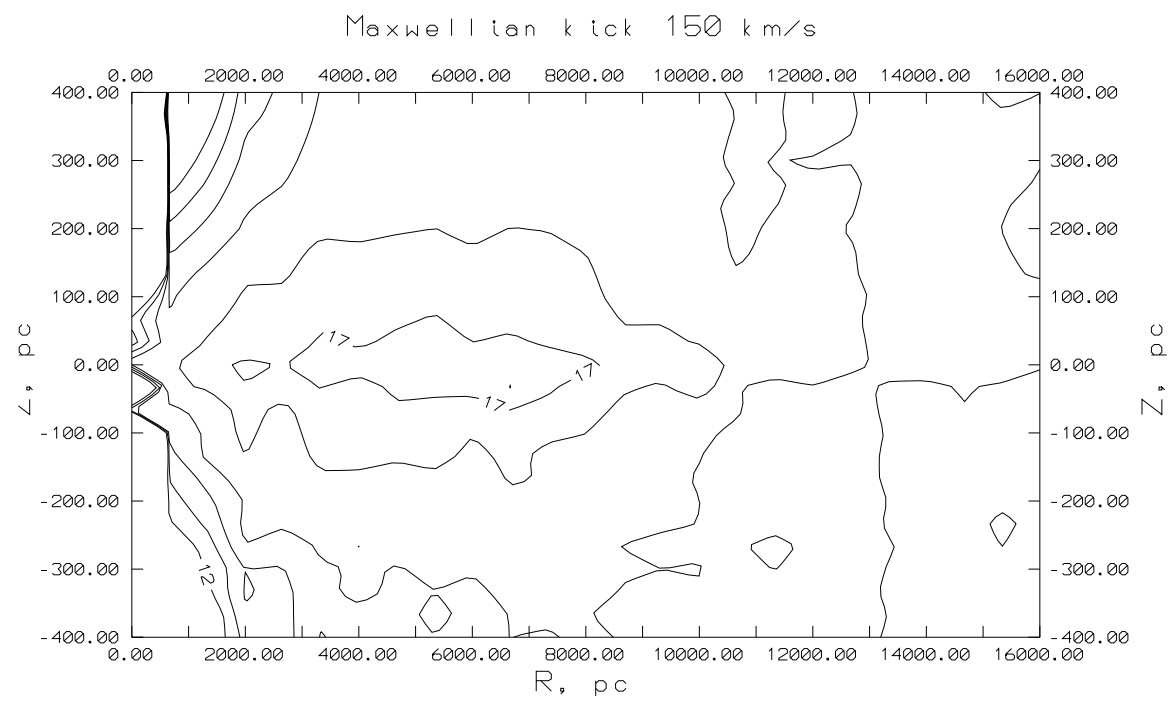

Figure 3: The luminosity distribution in R-Z plane for Maxwellian kick velocity $(150 \mathrm{~km} / \mathrm{s})$

$$
\begin{gathered}
n(R, Z)=n_{H I}+2 \cdot n_{H_{2}} \\
n_{H_{2}}=n_{0} \cdot \exp \left[\frac{-Z^{2}}{2 \cdot(70 p c)^{2}}\right]
\end{gathered}
$$

If $2 k p c \leq R \leq 3.4 k p c$, then

$$
n_{H I}=n_{0} \cdot \exp \left[\frac{-Z^{2}}{2 \cdot(140 p c \cdot R / 2 k p c)^{2}}\right],
$$

For $R \leq 2 k p c n(R, Z)$ was assumed to be constant:

$$
n(R<2 k p c, Z)=n(R=2 k p c, Z)
$$

Of course, it is not accurate, so for the very central part of the Galaxy our results are only a rough estimation.

If $3.4 k p c \leq R \leq 8.5 k p c$, then

$n_{H I}=0.345 \cdot \exp \left[\frac{-Z^{2}}{2 \cdot(212 p c)^{2}}\right]+0.107 \cdot \exp \left[\frac{-Z^{2}}{2 \cdot(530 p c)^{2}}\right]+0.064 \cdot \exp \left[\frac{-Z}{403 p c}\right]$

If $8.5 \leq R \leq 16 \mathrm{kpc}$, then 


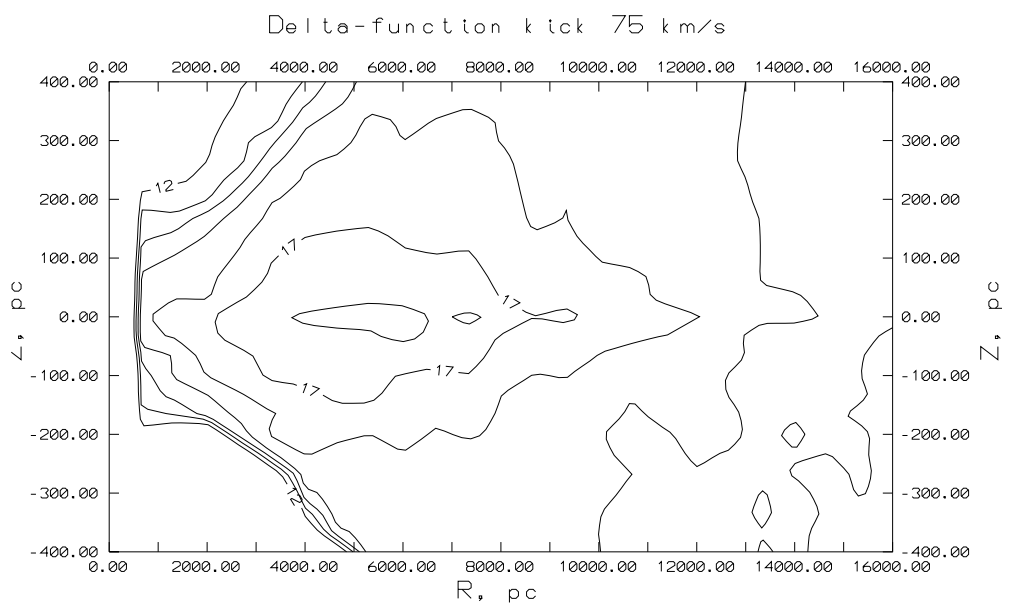

Figure 4: The luminosity distribution in R-Z plane for $\delta$-function kick velocity $(75 \mathrm{~km} / \mathrm{s})$

$$
n_{H I}=n_{\infty} \cdot \exp \left[\frac{-Z^{2}}{2 \cdot(530 p c \cdot R / 8.5 k p c)^{2}}\right]
$$

The density distribution is shown in the figure 1 .

Kick velocity was taken both: in the Maxwellian form with the maximum velocity $150 \mathrm{~km} / \mathrm{s}, 75 \mathrm{~km} / \mathrm{s}$ and $35 \mathrm{~km} / \mathrm{s}$ and as a $\delta$-function with $\mathrm{V}=150 \mathrm{~km} / \mathrm{s}$, $75 \mathrm{~km} / \mathrm{s}$ and $35 \mathrm{~km} / \mathrm{s}$ (see discussion in Lipunov et al. (1996)).

Sound velocity was taken to be $10 \mathrm{~km} / \mathrm{s}$. Luminosity was calculated using Bondi formula:

$$
L=\left(\frac{G M_{N S}}{R_{N S}}\right) 2 \pi\left(\frac{\left(G M_{N S}\right)^{2} n(R, Z)}{\left(V_{s}^{2}+V^{2}\right)^{3 / 2}}\right)
$$

\section{Results}

On the figures 2-7 we represent the results for two velocity distributions. On the figure 8 the slice at $\mathrm{Z}=+5 \mathrm{pc}$ for the maxwellian kick $\left(V_{\max }=150 \mathrm{~km} / \mathrm{s}\right)$ is shown.

As it is clearly seen from the figures, the distribution of the luminosity density (shown in arbitrary units) in R-Z plane forms a torus-like structure with the maximum at approximatelly $5 \mathrm{kpc}$. 


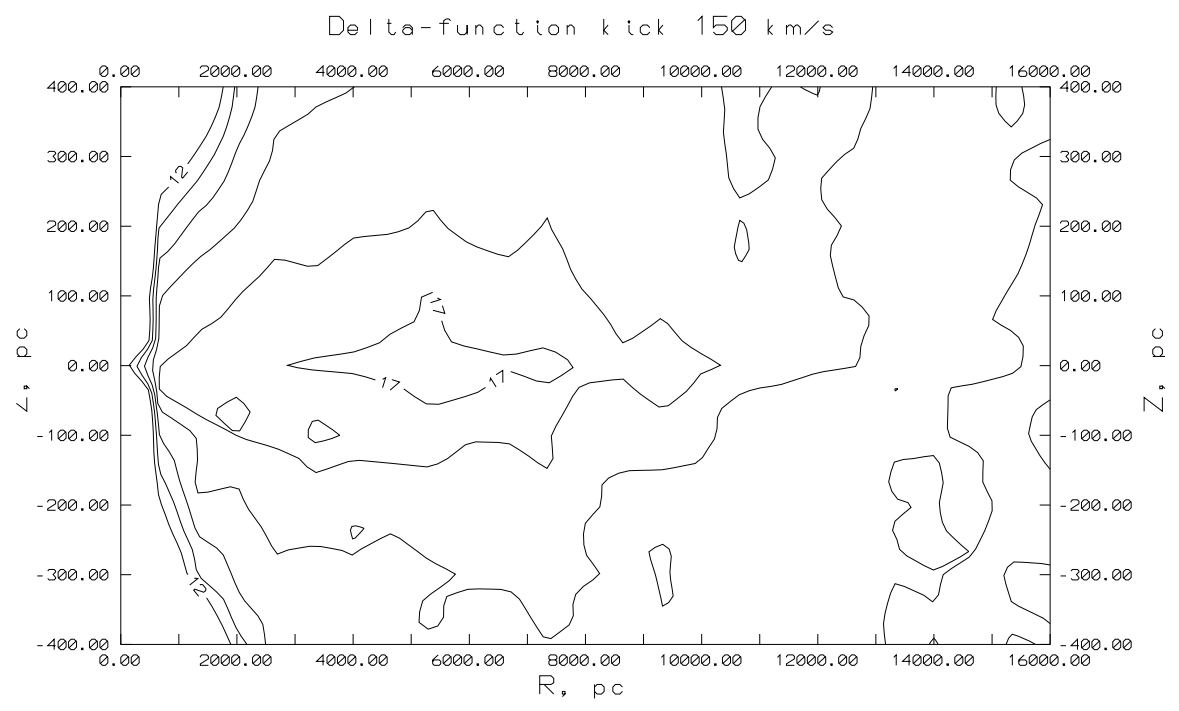

Figure 5: The luminosity distribution in R-Z plane for $\delta$-function kick velocity $(150 \mathrm{~km} / \mathrm{s})$

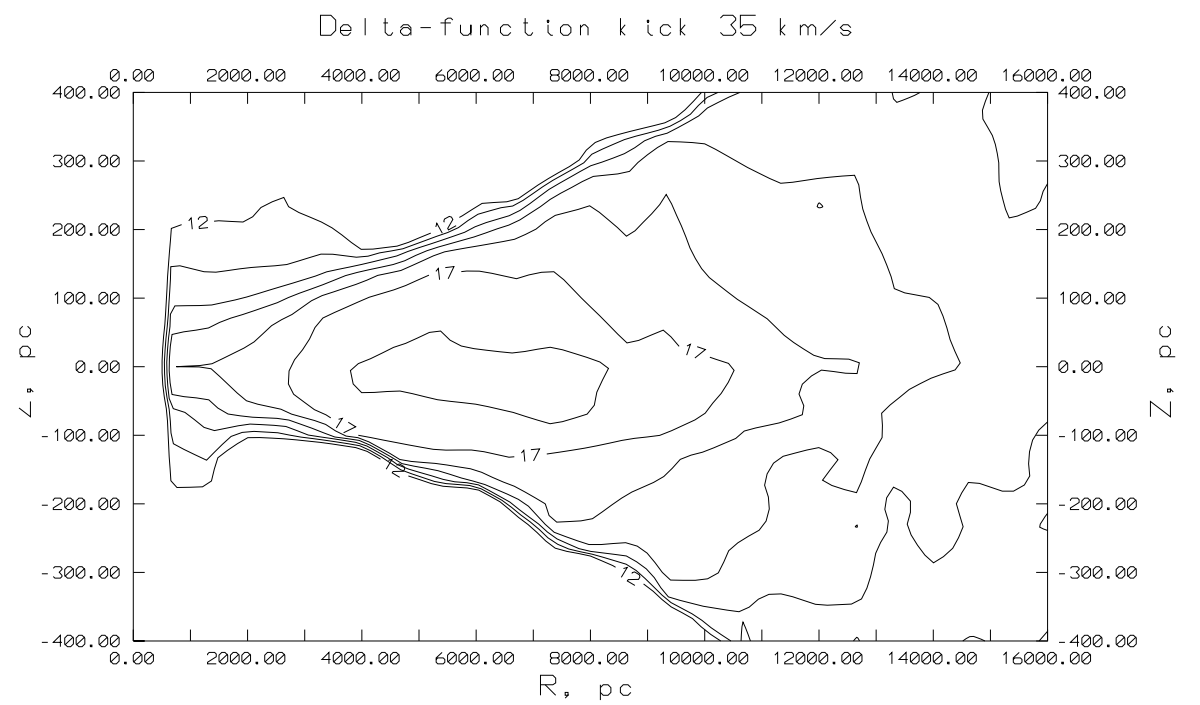

Figure 6: The luminosity distribution in R-Z plane for $\delta$-function kick velocity $(35 \mathrm{~km} / \mathrm{s})$ 


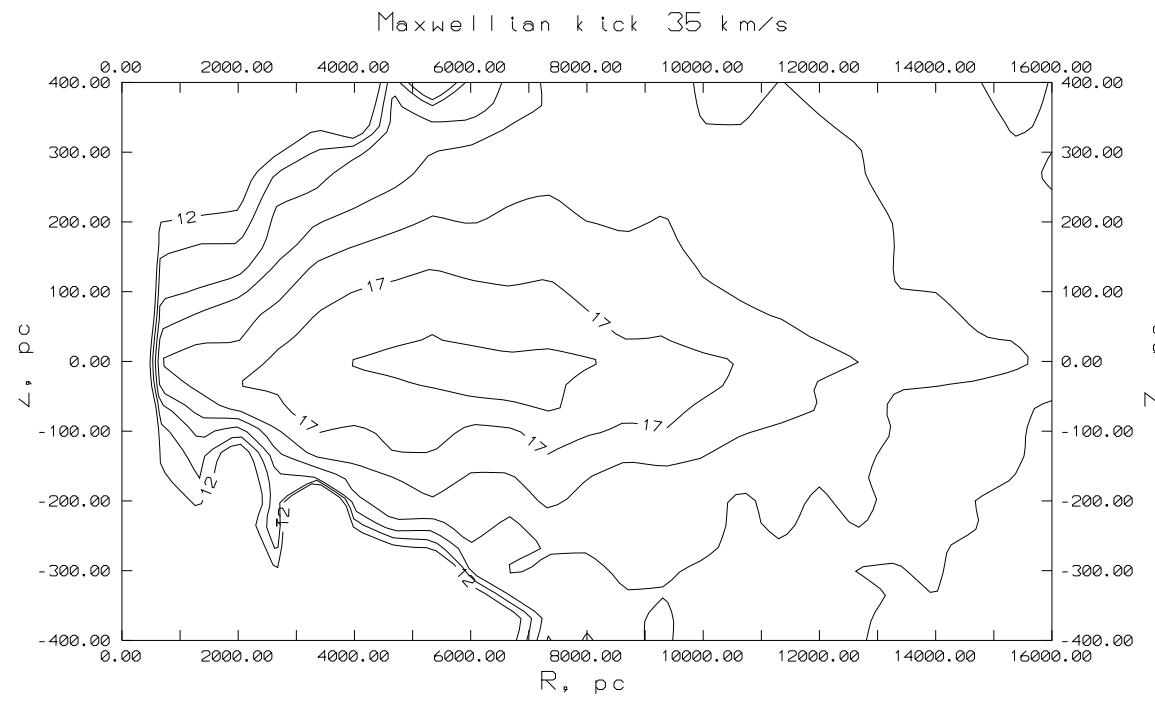

Figure 7: The luminosity distribution in R-Z plane for maxwellian kick velocity $(35 \mathrm{~km} / \mathrm{s})$

\section{Maxwellian kick $150 \mathrm{~km} / \mathrm{s}$}

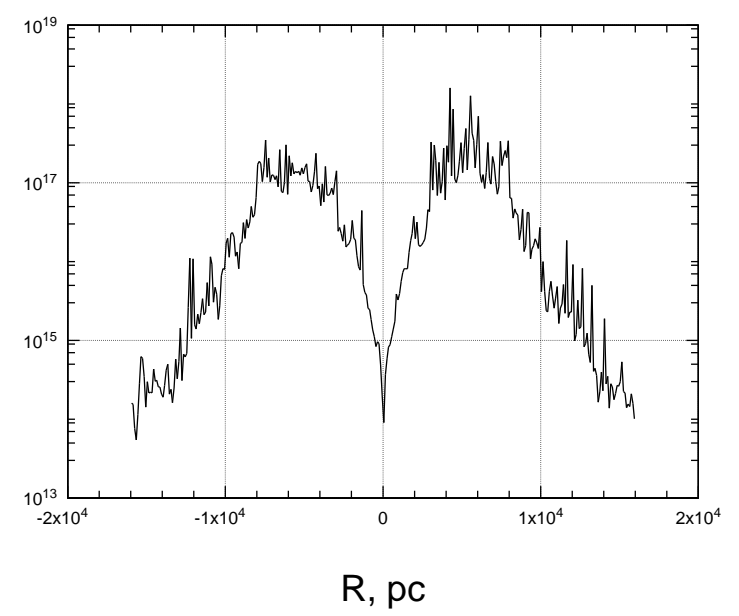

Figure 8: Slice at $\mathrm{Z}=+5 \mathrm{pc}$ for maxwellian kick velocity $(150 \mathrm{~km} / \mathrm{s})$ 


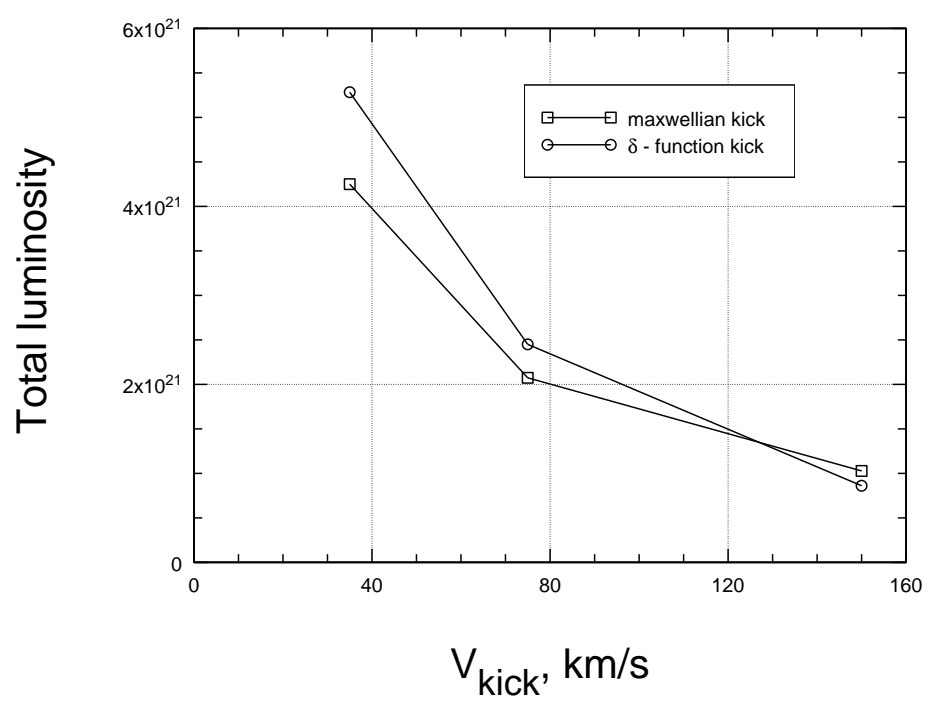

Figure 9: Total velocity (in arbitrary units) vs. kick velocity

\section{Discussion and concluding remarks}

The torus-like structure of that distribution is an interesting and important feature of the Galactic potential. Local maximums in the ISM distribution are smoothed (compare figures 1-7). As one can suppose, for low velocities we get greater luminosity. Stars with the Maxwellian distribution can penetrate deeper into the inner regions than stars with $\delta$-function velocity distribution (especially it is clear for high $\mathrm{Z}-200-400 \mathrm{pc}$ for low velocity distributions) because we for maxwellian kick we have both: more low velocity and more high velocity stars.

On fig.9 we show dependence of the total luminosity of the galaxy (in arbitrary units) from the kick velocity for two types of distributions. We mark very interesting feature: intersection of the curves at $\approx 125 \mathrm{~km} / \mathrm{s}$.

As me made very general assumptions, we argue, that such a distribution is not unique for our Galaxy, and all spiral galaxies must have such a distribution of the luminosity density, associated with accreting OINSs.

\section{Aknowledgements}

The work was supported by the RFFI (95-02-6053) and the INTAS (93-3364) grants. The work of S.P. was also supported by the ISSEP.

\section{References}


[1] N.G. Bochkarev, "Basics of the ISM physics", 1992, Moscow, Moscow State Univ. Press

[2] V.M. Lipunov and S.B. Popov, AZh, 71, 711, 1995

[3] V.M. Lipunov, K.A. Postnov and M.E. Prokhorov, A\&A, 310, 489, 1996

[4] B. Paczynski, ApJ 348, 485, 1990

[5] S.B. Popov, Astr. Circ., N1556, 1, 1994

[6] M.E. Prokhorov and K.A. Postnov, A\&A, 286, 437, 1994

[7] M.E. Prokhorov and K.A. Postnov, Astr. Astroph. Trans., 4, 81, 1993

[8] A. Treves and M. Colpi, A\&A, 241, 107, 1991

[9] S. Zane, R. Turolla, L. Zampieri, M. Colpi and A. Treves, ApJ, 1995, 451, 739 